04,03

\title{
Усиление и инверсия переменного электрического поля в мелкодисперсном диэлектрике
}

\section{(C) В.Ф. Харламов}

Приокский государственный университет, Орел, Россия

E-mail: vladkharl@rambler.ru

(Поступила в Редакцию 1 марта 2016 г.

В окончательной редакции 20 мая 2016 г.)

Установлено, что в двух плоскопараллельных слоях разных диэлектриков, заключенных между пластинами плоского конденсатора, происходит усиление более чем в $10^{3}$ раз синусоидального электрического поля. Для этого должны выполняться следующие условия: 1) один слой из двух образован мелкодисперсным диэлектриком, на поверхности частиц которого созданы ионизованные донорные центры, а в объеме частиц имеются свободные электроны; 2) диэлектрическая проницаемость порошка имеет отрицательное значение. При этом в слое порошка одновременно с усилением электрического поля происходит его инверсия.

Исследование выполнено при финансовой поддержке Министерства образования и науки РФ (грант № 479).

DOI: $10.21883 /$ FTT.2017.01.43948.75

\section{1. Введение}

Изучение электронных явлений в неоднородных полупроводниках привлекает внимание исследователей (см., например, [1]). В случае мелкодисперсных полупроводников, поверхность частиц которых легирована донорной примесью, волновые функции электронов зоны проводимости ограничены размерами зерен порошка. Вследствие этого электронные явления в таких мелкодисперсных материалах и однородных полупроводниках $n$-типа различаются существенным образом. Вольтамперные характеристики порошков, поверхность наночастиц которых насыщена межузельными атомами водорода (донор), содержат участки отрицательного дифференциального сопротивления, которое монотонно приближается к нулю при увеличении силы тока [2]. Согласно результатам теоретического анализа, полупроводниковые материалы, состоящие из сферических наночастиц, могут иметь термоэлектрическую эффективность, в десятки раз превышающую термоэлектрическую эффективность однородных полупроводников. Для этого термоэлектронная работа выхода частиц порошка должна быть менее $1 \mathrm{eV}$ [3], например в связи с наличием на их поверхности донорных центров.

Цель настоящей работы состоит в изучении поляризации в синусоидальном электрическом поле мелкодисперсных диэлектриков, на поверхности частиц которых созданы ионизованные донорные центры.

\section{2. Электрическое поле в двухслойных диэлектриках}

В слабом однородном переменном электрическом поле $E=E_{m} \exp (-i \omega t)$ диэлектрическая проницаемость однородного полупроводника $n$-типа равна [4]

$$
\begin{aligned}
\varepsilon & =\varepsilon^{\prime}+i \varepsilon^{\prime \prime}=\varepsilon_{c}+\frac{4 \pi i}{\omega} \sigma \\
& =\varepsilon_{c}^{\prime}-\frac{4 \pi}{\omega} \sigma^{\prime \prime}+i\left(\varepsilon_{c}^{\prime \prime}+\frac{4 \pi}{\omega} \sigma^{\prime}\right),
\end{aligned}
$$

где $\varepsilon^{\prime}$ и $\varepsilon^{\prime \prime}-$ ее вещественная и мнимая части, $\varepsilon_{c}=\varepsilon_{c}^{\prime}+i \varepsilon_{c}^{\prime \prime}-$ диэлектрическая проницаемость решетки без учета свободных носителей тока, $\sigma=\sigma^{\prime}+i \sigma^{\prime \prime}-$ электропроводность полупроводника, $\sigma^{\prime}$ и $\sigma^{\prime \prime}-$ вещественная и мнимая части электропроводности,

$$
\sigma^{\prime \prime}=-\frac{\omega e^{2}}{3} \int_{0}^{\infty} N(E) v^{2}(E) f_{0}^{\prime} \frac{\tau^{2}}{1+\omega^{2} \tau^{2}} d E
$$

$e$ - заряд электрона, $N(E)$ - плотность состояний в зоне проводимости, $E$ - энергия электрона, $v$ - его скорость, $f_{0}^{\prime}=\partial f_{0} / \partial E, f_{0}-$ распределение Ферми-Дирака $\left(f_{0}^{\prime}<0\right), \tau-$ время релаксации импульса электрона.

Используя для интеграла в выражении (2) теорему о среднем, с помощью формул (1) и (2) находим

$$
\varepsilon^{\prime}=\varepsilon_{c}^{\prime}-\frac{4 \pi}{\omega} \sigma^{\prime \prime}=\varepsilon_{c}^{\prime}-\frac{4 \pi e^{2} n}{m}\left\langle\frac{\tau^{2}}{1+\omega^{2} \tau^{2}}\right\rangle,
$$

где $n$ - концентрация свободных электронов, $m$ эффективная масса электропроводности [4],

$$
m=-3 \int_{0}^{\infty} N(E) f_{0} d E\left[\int_{0}^{\infty} N(E) v^{2} f_{0}^{\prime} d E\right]^{-1}
$$

Согласно (3), величина $\varepsilon^{\prime}$ может оказаться отрицательной [4]. Используя в качестве примера значения 
$\varepsilon_{c}^{\prime}=14, n=7.98 \cdot 10^{19} \mathrm{~m}^{-3},\left\langle\tau^{2}\right\rangle=10^{-24} \mathrm{~s}^{2}, \quad m=10^{-31} \mathrm{~kg}$, $0<\omega \ll 1 / \tau$, с помощью выражения (3) получаем $\varepsilon^{\prime}=-15$.

В случае мелкодисперсного диэлектрика, поверхность частиц которого легирована донором, вещественная часть электропроводности $\sigma^{\prime}$ мала из-за относительно небольшой площади контактов между частицами порошка. В этом случае в выражении (1) величина $4 \pi \sigma^{\prime} / \omega$ мала. Поэтому, пренебрегая мнимой частью $\varepsilon$, для ее среднего по объему диэлектрика значения находим

$$
\varepsilon \approx \varepsilon^{\prime} \approx \varepsilon_{c}^{\prime}-\frac{4 \pi}{\omega} \sigma^{\prime \prime} \approx \varepsilon_{c}^{\prime}-\frac{4 \pi e^{2} n}{m}\left\langle\frac{\tau^{2}}{1+\omega^{2} \tau^{2}}\right\rangle,
$$

где $\sigma^{\prime \prime} \gg \sigma^{\prime}, \omega_{l}>\omega \gg 4 \pi \sigma^{\prime} / \varepsilon_{c}^{\prime}, \omega_{l}-$ минимальная частота переменного поля, поглощаемого кристаллической решеткой. Согласно (4), диэлектрическая проницаемость мелкодисперсного диэлектрика, на поверхности частиц которого созданы донорные центры, может оказаться отрицательной. При этом $\varepsilon$ не зависит от $\omega$ явно при выполнении условий

$$
4 \pi \sigma^{\prime} / \varepsilon_{c}^{\prime} \ll \omega \ll \tau^{-1} .
$$

Пусть между параллельными пластинами плоского конденсатора находятся два плоскопараллельных слоя разных диэлектриков толщиной $l$ и $l_{1}$. Используем выражения

$$
U+U_{1}=U_{0}, \varepsilon E=\varepsilon_{1} E_{1}, E l+E_{1} l_{1}=U_{0},
$$

где $U_{0}, U$ и $U_{1}$ - электрические напряжения на конденсаторе и слоях диэлектрика, $\varepsilon$ и $\varepsilon_{1}-$ диэлектрические проницаемости этих диэлектриков, $E$ и $E_{1}-$ напряженности электрического поля в них. С помощью выражений (5) находим

$$
\begin{gathered}
E=\frac{U_{0}}{l \delta}, E_{1}=\frac{\varepsilon E}{\varepsilon_{1}}, \\
\delta=1+\frac{\varepsilon l_{1}}{\varepsilon_{1} l} .
\end{gathered}
$$

Пусть выполняются условия

$$
\varepsilon<0, \quad \varepsilon_{1}>0, \quad|\delta| \ll 1, \quad l \neq 0, \quad l_{1} \neq 0 .
$$

Выражения (5)-(8) описывают эффект усиления электрического поля в двухслойных диэлектриках. Этот эффект невозможен в однослойном диэлектрике, поскольку, полагая $l_{1}=0$, с помощью формул (6) и (7) находим

$$
\delta=1, \quad E=\frac{U_{0}}{l} .
$$

Изменение свободной энергии $F$ диэлектрика вследствие его поляризации внешним электрическим полем $E_{0}$ при постоянных температуре и объеме равно энергии электрического поля в диэлектрике [5]

$$
\Delta F=\frac{\varepsilon E^{2}}{8 \pi}
$$

где $\varepsilon-$ его диэлектрическая проницаемость. Используя выражения (6),(7) и (10), в случае выполнения условий (8) находим, что в результате создания электрического поля в двухслойном диэлектрике его свободная энергия изменяется на величину

$\Delta F_{\Sigma}=\Delta F+\Delta F_{1}=\frac{\varepsilon E^{2} S_{p} l}{8 \pi}+\frac{\varepsilon_{1} E_{1}^{2} S_{p} l_{1}}{8 \pi}=\frac{\varepsilon E^{2} V \delta}{8 \pi}=\delta \Delta F$,

где $\Delta F_{\Sigma}>0$ - работа источника поля, $\Delta F$ и $\Delta F_{1}-$ изменение свободной энергии слоев диэлектриков, $\varepsilon<0$, $\Delta F<0, \quad \delta<0, \quad|\Delta F| \leq F_{0}, \quad F_{0}=U-T S-$ свободная энергия порошка без поля, $V=S_{p} l-$ объем слоя порошка, $S_{p}$ - площадь его поверхности. На основании выражений (10) и (11) имеем

$$
E= \pm\left(\frac{8 \pi \Delta F_{\Sigma}}{\varepsilon V \delta}\right)^{1 / 2}
$$

Приравняем правые части (12) и первого выражения в (6). Получаем

$$
\delta=\frac{\varepsilon V U_{0}^{2}}{8 \pi \Delta F_{\Sigma} l^{2}} .
$$

Подставляя (13) в (12), находим

$$
E= \pm \frac{8 \pi \Delta F_{\Sigma}}{\varepsilon S_{p} U_{0}}=\frac{4 \pi \sigma_{p}}{\varepsilon},
$$

где $\Delta F_{\Sigma}=\sigma_{p} S_{p} U_{0} / 2 ; \sigma_{p}-$ поверхностная плотность поляризационных зарядов на межфазных границах. Согласно (13) и (14), численные значения $\delta$ и $E$ зависят от параметров системы и работы внешнего поля $\Delta F_{\Sigma}$.

Рассмотрим замкнутую электрическую цепь, состоящую из источника переменного напряжения $u_{x}=u_{x m} \sin \omega t$ и емкостного ,делителя“ из последовательно соединенных „измерительного“ и „эталонного“ конденсаторов с электрическими емкостями $c_{t}$ и $c_{y}$ соответственно. Поскольку возможны два взаимно противоположных направления вектора $E$ (см. (14)), полярность напряжения на измерительном конденсаторе $U_{0}= \pm E l \delta$ (см. (6)) может совпадать или не совпадать с полярностью напряжения на эталонном конденсаторе: $u_{y}=u_{x} \pm U_{0}$. Ток в цепи равен

$$
J=J_{m} \sin \omega t,
$$

где $J_{m}=u_{x m} \omega C_{x}, C_{x}=c_{t} c_{y} /\left(c_{t}+c_{y}\right)$. С помощью этих выражений получаем $u_{y}=u_{y m} \sin \omega t$,

$$
u_{y m}=\frac{J_{m}}{\omega c_{y}}=\frac{c_{t}}{c_{t} \pm c_{y}} u_{x m} \equiv g u_{x m},
$$

где $u_{x m}$ и $u_{y m}$ - амплитуды напряжения на делителе и эталонном конденсаторе соответственно. 


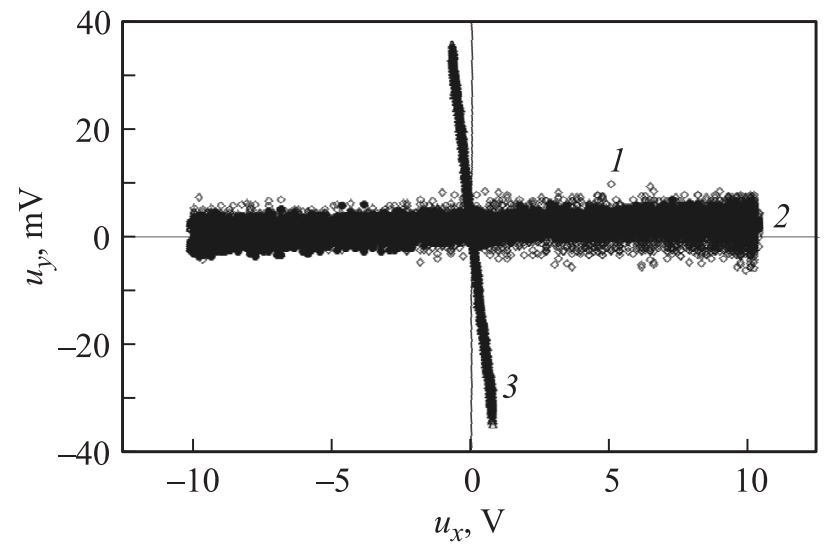

Петли диэлектрического гистерезиса для слоя мелкодисперсного перманганата калия, помещенного между пластинами диэлектрика в атмосферу воздуха (1), в вакуум (2) и в среду водорода с давлением $P_{\mathrm{H}}=3 \cdot 10^{4} \mathrm{~Pa}(3) . T=23^{\circ} \mathrm{C}$, $v=100 \mathrm{~Hz}, \quad c_{y}=320 \mathrm{nF}$. Значения $u_{y}$ для кривых 1 и 2 увеличены в 52 раза.

\section{3. Экспериментальные результаты и их обсуждение}

В экспериментах использовались проницаемые для молекул газов мелкодисперсные соединения $\mathrm{KMnO}_{4}$ марки хч и $\mathrm{Al}_{2} \mathrm{O}_{3}$. Средний размер частиц перманганата калия в форме параллелепипеда равен $14 \mu \mathrm{m}$. Частицы $\mathrm{Al}_{2} \mathrm{O}_{3}$ имели форму шара со средним диаметром $100 \mathrm{~nm}$. Слой порошка (далее - образец) толщиной $l \approx 0.2 \mathrm{~mm}$ и площадью $S_{p} \approx 50 \mathrm{~mm}^{2}$ помещался между двумя плоскопараллельными пластинками слюды с суммарной толщиной $l_{1}=0.08 \mathrm{~mm}$, находящимися в контакте с плоскими металлическими электродами. Этот измерительный конденсатор с образцом помещался в вакуумную камеру из стекла. В камеру после ее откачки напускался водород с давлением $P_{\mathrm{H}}=3 \cdot 10^{4} \mathrm{~Pa}$. Чистота водорода составляла $99.995 \%$. Установка экранировалась от электромагнитных помех.

С использованием метода Сойера-Тауэра (см., например, [6,7]) на цифровом осциллографе Hantek DSO 2150 USB измерялись петли диэлектрического гистерезиса образцов на частотах $v=20-10^{3} \mathrm{~Hz}$. Для этого использовался емкостный делитель напряжения в виде последовательно соединенных измерительного и эталонного конденсаторов. К нему подключался генератор синусоидального напряжения амплитудой $10 \mathrm{~V}$. Синусоидальные напряжения на делителе $u_{x}$ и эталонном конденсаторе $u_{y}$ подавались соответственно на горизонтальные и вертикальные пластины осциллографа.

В случае измерительного конденсатора со слоем мелкодисперсного перманганата калия форма петель диэлектрического гистерезиса зависит от газовой среды. В воздухе и вакууме петли диэлектрического гистерезиса имеют форму отрезка прямой с незначительным положительным наклоном (кривые 1 и 2 на рисунке), что соответствует отсутствию носителей тока в частицах порошка. После напуска водорода с давлением
$P_{\mathrm{H}}=3 \cdot 10^{4} \mathrm{~Pa}$ в вакуумную камеру с образцом $\mathrm{KMnO}_{4}$ происходит самопроизвольное уменьшение амплитуды напряжения $u_{x}$ и увеличение амплитуды напряжения $u_{y}$ в $10^{3}-10^{4}$ раз. При этом петли диэлектрического гистерезиса имеют форму отрезка прямой с отрицательным наклоном (кривая 3 на рисунке). Угол отрицательного наклона и величина отрезков прямых $u_{y}=g u_{x}$, (где $g<0$ - коэффициент) слабо зависят от частоты синусоидального напряжения. Площадь петель диэлектрического гистерезиса равна нулю.

В тех же условиях эффект скачкообразного самопроизвольного изменения величин $u_{x m}$ и $u_{y m}$ после напуска водорода в откачанную камеру с образцом не наблюдается, если а) между пластинами слюды помещен слой мелкодисперсного оксида алюминия или b) слой порошка $\mathrm{KMnO}_{4}$ либо $\mathrm{Al}_{2} \mathrm{O}_{3}$ находится в непосредственном контакте с электродами $\left(l_{1}=0\right)$.

В этих двух случаях независимо от среды для порошка (вакуум, воздух или водород) петли диэлектрического гистерезиса имеют форму эллипса с положительным наклоном. Площадь этих петель зависит от частоты переменного напряжения.

Перейдем к обсуждению экспериментальных данных. После напуска водорода в откачанную камеру с образцом $\mathrm{KMnO}_{4}$ резкому увеличению амплитуды $u_{y m}$ и появлению зависимости $u_{y}=g u_{x}$, где $g<0$ (ср. кривые 3 и 1,2 на рисунке) соответствуют выражения (6), (7), (13)-(15), в которых $\varepsilon<0,|\delta| \ll 1, g \approx-c_{t} / c_{y}$. Согласно (4), выполнение условия $\varepsilon<0$ обусловлено появлением свободных электронов в частицах порошка, которые возникают благодаря диссоциативной хемосорбции молекул водорода. При этом атомы водорода, занимая положение в междоузлиях кристаллической решетки, образуют в запрещенной зоне полупроводника ионизованные донорные уровни [4]. Условия (9) соответствуют экспериментальным данным, согласно которым эффект усиления электрического поля в слое порошка отсутствует, если он находится в контакте с электродами $\left(l_{1}=0\right)$.

Используя значения $\varepsilon_{1}=6$ (для слюды), $l=0.2 \mathrm{~mm}$, $l_{1}=0.08 \mathrm{~mm}, \delta \ll 1$ и формулу (7), получаем $\varepsilon \approx-15$. С помощью прямой 3 (см. рисунок) находим $u_{x m}=0.8 \mathrm{~V}$, $u_{y m}=36 \mathrm{mV}$. До напуска водорода в вакуумную камеру $\varepsilon=\varepsilon_{v} \approx 14$ и при $u_{x v}=0.8$ (кривая 3) $u_{y v} \approx 10^{-5} \mathrm{~V}$. Используем выражения $c_{y} u_{y m}=\sigma_{p m} S_{p}$, $E_{m}=4 \pi \sigma_{p m} / \varepsilon=4 \pi c_{y} u_{y m} /\left(\varepsilon S_{p}\right)$. После помещения слоя порошка двухслойной структуры в среду водорода напряженность поля в этом слое увеличивается в $E_{m} / E_{v}=\varepsilon_{v} u_{y m} /\left(\varepsilon u_{y v}\right) \approx 4 \cdot 10^{3}$ раз по сравнению с наблюдаемой при его нахождении в вакууме или в атмосфере воздуха.

Хемосорбция водорода на поверхности частиц оксида алюминия не сопровождается появлением поверхностных носителей тока. Поэтому в тех же условиях для слоя частиц $\mathrm{Al}_{2} \mathrm{O}_{3}$ неравенство $\varepsilon<0$ не выполняется, сопутствующие эффекты усиления и инверсии поля не наблюдаются.

Автор выражает благодарность А.А. Соколову за помощь в работе. 


\section{Список литературы}

[1] Н.А. Тулина. УФН 177, 1231 (2007).

[2] В.Ф. Харламов, Д.А. Коростелёв, И.Г. Богораз, О.А. Миловидова. ФТТ 54, 1204 (2012).

[3] В.Ф. Харламов. ЖЭТФ 144, 97 (2013).

[4] В.Л. Бонч-Бруевич, С.Г. Калашников. Физика полупроводников. Наука, М. (1990). С. 449.

[5] И.П. Базаров. Термодинамика. Высш. шк., М. (1991). С. 190.

[6] И.П. Пронин, Е.Ю. Каптелов, Е.А. Тараканов, Т.А. Шаплыгина, В.П. Афанасьев, А.В. Панкрашкин. ФТТ 44, 739 (2002).

[7] W.L. Warren, B.A. Tuttle, D. Dimos, G.E. Pike, H.N. AlShareef, R. Ramesh, J.T. Evans. Jpn. J. Appl. Phys. 35 (Pt 1), 1521 (1996). 